\title{
All-Cause Mortality Risk with Direct Oral Anticoagulants and Warfarin in the Primary Treatment of Venous Thromboembolism
}

\author{
Richard F. MacLehose ${ }^{3}$ \\ ${ }^{1}$ Chronic Disease Research Group, Hennepin Healthcare Research \\ Institute, Minneapolis, Minnesota, United States \\ 2 OptumLabs Visiting Fellow, Cambridge, Massachusetts, \\ United States \\ ${ }^{3}$ Division of Epidemiology and Community Health, School of Public Health, \\ University of Minnesota, Minneapolis, Minnesota, United States \\ ${ }^{4}$ Department of Medicine, Larner College of Medicine, University of \\ Vermont, Burlington, Vermont, United States \\ ${ }^{5}$ Department of Pathology and Laboratory Medicine, Larner College of \\ Medicine, University of Vermont, Burlington, Vermont, United States \\ ${ }^{6}$ Department of Epidemiology, Rollins School of Public Health, Emory \\ University, Atlanta, Georgia, United States \\ ${ }^{7}$ College of Pharmacy, University of Minnesota, Minneapolis, \\ Minnesota, United States \\ 8 Institute for Health Informatics, University of Minnesota, \\ Minneapolis, Minnesota, United States
}

Nicholas S. Roetker ${ }^{1,2,3}$ Pamela L. Lutsey ${ }^{3}$ Neil A. Zakai ${ }^{4,5}$ Alvaro Alonso ${ }^{6}$ Terrence J. Adam ${ }^{7,8}$

\author{
Address for correspondence Nicholas S. Roetker, PhD, MS, Chronic \\ Disease Research Group, Hennepin Healthcare Research Institute, 701 \\ Park Avenue, Suite S2.100, Minneapolis, MN 55415, United States \\ (e-mail: nroetker@cdrg.org).
}

Thromb Haemost 2018;118:1637-1645.

\begin{abstract}
Keywords

- deep vein thrombosis

- pulmonary embolism

- epidemiologic studies

- management of disease

Oral anticoagulants used for the primary treatment of venous thromboembolism (VTE) include warfarin and the more recently introduced direct oral anticoagulants (DOACs), including rivaroxaban, apixaban, dabigatran and edoxaban. Information on the comparative safety of these medications in routine clinical practice is lacking. We identified patients with diagnoses for VTE and prescriptions for oral anticoagulants using claims data from a large U.S. insurance database from 2012 to 2017. Marginal structural logistic models were used to examine associations between type of oral anticoagulant and risk of all-cause mortality. Of 62,431 enrolees in this analysis, $51 \%$ were female and the mean age was 61.9 years. Initial oral anticoagulant prescriptions were for warfarin $(n=35,704)$, rivaroxaban $(n=21,064)$ and apixaban $(n=5,663)$. A total of 1,791 deaths occurred within 6 months of the initial oral anticoagulant prescription. Risk of all-cause mortality was not associated with having a prescription for warfarin versus any DOAC or between any head-to-head DOAC comparisons. Also, associations generally did not vary when stratified by VTE type, sex, age, co-morbidities (including renal disease) or anti-platelet medication use. In this observational study, the associations with all-cause mortality comparing DOACs versus warfarin agree with results from previous clinical trials and observational studies, while the associations for headto-head DOAC comparisons provide new information on the comparative safety of DOACs. Our findings suggest that other criteria such as patient preference, cost, recurrent VTE risk or bleeding risk should be used when determining the choice of anticoagulant for the primary treatment of VTE.
\end{abstract}

received

May 16, 2018

accepted after revision

July 5, 2018 (c) 2018 Georg Thieme Verlag KG Stuttgart · New York
DOI https://doi.org/

$10.1055 / \mathrm{s}-0038-1668521$. ISSN 0340-6245. 


\section{Introduction}

Evidence from phase III clinical trials demonstrates that during the primary treatment of venous thromboembolism (VTE) (i.e., first 3-6 months after the event), direct oral anticoagulants (DOACs; dabigatran, rivaroxaban, apixaban and edoxaban) are non-inferior to vitamin $\mathrm{K}$ antagonist (VKA) therapy for preventing recurrent, symptomatic VTE and VTE-related death. ${ }^{1-6}$ However, results from clinical trials may have limitations generalizing to routine clinical practice. ${ }^{7}$ Also, trials of new drugs are often underpowered to detect differences in risk for important safety outcomes, such as all-cause mortality. Indeed, the 10th Edition Antithrombotic Guidelines by the American College of Chest Physicians rates the quality of evidence for each DOAC versus VKA therapy for the prevention of all-cause mortality as 'moderate' due to lack of precision. ${ }^{8}$

For these reasons, observational cohort studies have recently begun to assess the comparative safety and effectiveness of DOACs versus VKA therapy in the community-wide setting. ${ }^{9-13}$ Two studies compared the safety of rivaroxaban versus VKA therapy with respect to all-cause mortality, although the number of events was relatively small $(\leq 125$ total deaths in each study); ${ }^{11,12}$ another study had a larger number of deaths, but made a pooled comparison of any DOAC (rivaroxaban, apixaban or dabigatran) versus VKA therapy. ${ }^{13}$ We are unaware of any observational studies of the primary treatment of VTE with all-cause mortality as an outcome that have compared the use of apixaban separately versus VKA therapy or compared DOACs in a head-to-head fashion.

In this study, we used administrative claims data from a large U.S. insurance database to compare the relative risk (RR) of all-cause mortality between patients with prescriptions for rivaroxaban, apixaban and VKA therapy (warfarin) for the primary treatment of VTE.

\section{Materials and Methods}

\section{Study Population and Exposure Ascertainment}

This study involves a retrospective analysis of claims data from OptumLabs ${ }^{\circledR}$ Data Warehouse, which includes de-identified claims data for privately insured and Medicare Advantage enrolees in a large, private, U.S. health plan. The database contains longitudinal health information on enrolees, representing a diverse mixture of ages, races/ethnicities and geographic regions across the United States. The health plan provides comprehensive full insurance coverage for physician, hospital and prescription drug services. ${ }^{14}$ All enrolee data available for research is pre-existing and de-identified in compliance with the Health Insurance Portability and Accountability Act of 1996 and hence is exempted from institutional review board approval.

We identified a cohort of enrolees between 2 November 2012 and 31 March 2017 who had (1) at least 1 inpatient, 1 emergency room or 2 outpatient (separated by at least a week but less than a year) claims for VTE and (2) at least 1 pharmacy claim for an oral anticoagulant (warfarin, dabigatran, rivaroxaban, apixaban or edoxaban) filled within 7 days before or 31 days after the initial VTE diagnosis. Because of small numbers, patients using dabigatran or edoxaban were not included in this study. The date of first anticoagulant prescription falling within the specified time range around the VTE claim was defined as the index date. The time window of interest began on 2 November 2012 because this was the date of Food and Drug Administration approval of rivaroxaban for the treatment of VTE. VTE cases were identified based on the International Classification of Diseases, Ninth Revision, Clinical Modification (ICD-9-CM) or ICD-10-CM diagnosis codes shown in -Supplementary Table S1 (available in the online version). The positive predictive value of VTE cases identified using the list of ICD-9-CM claims is roughly 75 to $85 \%{ }^{15-18}$ and is higher when additionally requiring an anticoagulant pharmacy claim. ${ }^{19,20}$ The validity for identifying warfarin users via pharmacy claims is excellent (sensitivity: 94\%, positive predictive value: $99 \%) .{ }^{21}$ Presently, no validation data exist on the accuracy of DOAC claims, but accuracy should be comparable.

\section{Outcome Ascertainment}

The outcome of interest, all-cause mortality, was ascertained based on two data sources. First, enrolees in the cohort were linked with the Social Security Administration (SSA) Death Master File, allowing the capture of both out-of-hospital and in-hospital deaths. Linkage to the SSA Death Master File can be based on several identifying criteria, including name, date of birth and Social Security number. For this analysis, we required a match based on Social Security number to consider death present. Historically, the mortality information in the SSA Death Master File had adequate sensitivity (86\%) and high specificity (100\%). ${ }^{22}$ However, since 2011 death information from state records has not been included in the SSA Death Master File, which is known to be now missing approximately $40 \%$ of deaths. ${ }^{22}$ Therefore, we additionally used medical claims with a discharge status of 'expired' as a supplemental means of identifying in-hospital deaths.

\section{Covariates}

All covariates of interest were measured using information from up to 180 days of enrolment prior to and including the index date. Age and sex were determined from the medical or pharmacy claims on the index date. Geographic region (U.S. census region) was ascertained from insurance member coverage records. Co-morbid disease, frailty and concomitant medications are potentially important confounders to consider since they may influence treatment choice and mortality. Malignancy, renal disease, liver disease, obesity, coagulopathy and numerous other constituent variables of the Charlson and Elixhauser co-morbidity indices were selected as covariates based on pre-defined ICD-9-CM and ICD-10-CM diagnosis codes. ${ }^{23}$ We also defined variables related to clinical manifestations of frailty, including abnormal gait, cachexia and use of durable medical equipment, based on pre-defined diagnosis and procedure codes. ${ }^{24}$ See - Supplementary Table S2 (available in the online version) for a full list of the co-morbidity and frailty variables included in this analysis. Pharmacy claims were used to identify concomitant or recent use of medications, 
including insulin, anti-diabetics, anti-hypertensives, statins, anti-platelets, celecoxib, narcotic analgesics and anti-neoplastics. Since aspirin is widely used over-the-counter, it was not included among the group of anti-platelet medications.

\section{Exclusions}

We made several exclusions due to having missing covariates or for other reasons. In particular, we excluded enrolees with a history of VTE due to the differing treatment recommendations for those with recurrent VTE. Similarly, including prevalent DOAC or warfarin users in the analysis may lead to bias, ${ }^{25}$ so we excluded any participants with a prescription claim for an oral anticoagulant prior to the index date. Of the 93,999 enrolees identified as candidates for inclusion in the study, we excluded those who were less than 18 years old or whose sex or geographic region was unknown or missing $(n=1,432)$, those who had a history of VTE or a previous pharmacy claim for an oral anticoagulant $(n=13,557)$ and those with fewer than 90 days of continuous health plan enrolment prior to the index date $(n=15,944)$.

\section{Statistical Analysis}

Baseline participant characteristics were presented by treatment group. All-cause deaths occurring by 3 and 6 months after the index date were identified. For the primary analysis, we used logistic regression to estimate marginal odds ratios (ORs) and 95\% confidence intervals (CIs) for the association between oral anticoagulant use and all-cause mortality for six different comparisons: rivaroxaban versus warfarin, apixaban versus warfarin and apixaban versus rivaroxaban. For analyses involving apixaban, all enrolees with an index date before the date of the drug's approval for treating VTE (21 August 2014) were excluded. To mimic the intention-to-treat design of the randomized trials comparing DOACs to warfarin, we analysed enrolees according to the oral anticoagulant used at the index date regardless of whether they discontinued during followup, as has been done previously. ${ }^{26}$

For every comparison, we fit two sets of models: unadjusted (crude) logistic regression and inverse-probability-of-treatment weighted (IPTW) marginal structural logistic models. ${ }^{27}$ For the latter models, stabilized weights were calculated as the inverse of the conditional predicted probability of being prescribed the oral anticoagulant that was actually prescribed multiplied by the marginal prevalence of that oral anticoagulant. The conditional predicted probability of using an oral anticoagulant was estimated using logistic regression, in which the log-odds of using an oral anticoagulant was modelled as a function of age, age, ${ }^{2}$ sex, U.S. census region, index date, index date, ${ }^{2}$ diagnosis setting (inpatient or emergency room vs. outpatient), VTE type (pulmonary embolism [PE] vs. deep vein thrombosis [DVT] alone), the constituent co-morbidity and frailty variables and concomitant medications. We refer to this model as the exposure model. If certain anticoagulants are associated with a higher risk of out-of-hospital mortality, this may lead to differential outcome misclassification. ${ }^{28,29}$ So, as a sensitivity analysis, we estimated the associations for the six main comparisons using only the Death Master File data for the all-cause mortality outcome.
Because clinical trials and other observational studies have compared the risk of mortality for rivaroxaban and apixaban versus VKA therapy, we summarized these previous results with those from this study using inverse variance-weighted random effects meta-analysis. For the comparison of rivaroxaban versus VKA therapy, results are stratified by DVT/PE, in line with the design of the EINSTEIN trials. ${ }^{2,3}$

We also estimated the association between oral anticoagulant use and all-cause mortality using IPTW marginal structural logistic models stratified by specific covariates of interest, including by VTE type, sex, age, renal disease, liver disease, overweight and obesity and use of anti-platelet medications. We tested for evidence of multiplicative interaction on the log OR scale by including a cross-product term between the covariate and anticoagulant treatment. We also estimated the relative excess risk due to interaction (RERI) and $95 \%$ CIs as a measure of additive interaction, using the delta method to estimate standard errors, ${ }^{30}$ since the additive scale may be the most important from a biological and public health perspective. ${ }^{31}$ A super-additive (sub-additive) interaction, indicated by a RERI greater than (less than) than 0 , means that the combined effect of the two exposures is larger (smaller) than the sum of the individual effects. Statistical analyses were performed using SAS, version 9.4 (SAS Institute, Cary, North Carolina, United States).

\section{Results}

The analyses that follow are based on a total of 62,431 enrolees diagnosed with VTE and prescribed an oral anticoagulant. The sample was $51 \%$ female, and the mean age was 61.9 years with a range from 18 to 89 years. Due to the small number of individuals older than 89 years, participants aged $>89$ years were set to a value of 89 to preserve protected health information. The numbers of prescriptions for the initial oral anticoagulant were 35,704 for warfarin, 21,064 for rivaroxaban and 5,663 for apixaban. Because of small numbers, we did not include enrolees with an initial prescription for dabigatran $(n=509)$ or edoxaban $(n=19)$ in any subsequent analyses (there were 12 deaths in the dabigatran group and 0 deaths in the edoxaban group occurring within 6 months). When comparing warfarin versus DOAC users, warfarin users on average were older and had a higher prevalence of co-morbidities and concomitant medication use (-Table 1). For comparisons involving head-to-head comparisons of DOACs, apixaban users on average were the oldest and had the highest prevalence of co-morbidities, while rivaroxaban users on average were the youngest and had the lowest co-morbidity burden (-Supplementary Table 53, available in the online version). Among the 62,431 enrolees included in the analysis, we identified 1,213 (1.9\%) all-cause deaths occurring within 3 months and 1,791 (2.9\%) all-cause deaths occurring within and 6 months of the index date. Compared with warfarin users, the risk of all-cause mortality within 6 months was lower among rivaroxaban users $(\mathrm{OR}, 0.59,95 \% \mathrm{CI}, 0.53,0.66)$ and apixaban users (OR, 0.81, 95\% CI, 0.68, 0.97) in crude models (- Table 2). In IPTW models adjusting for demographics, co-morbidities and 
Table 1 Patient characteristics

\begin{tabular}{|c|c|c|c|c|}
\hline & \multicolumn{2}{|c|}{$\begin{array}{l}\text { Comparison } 1 \\
\text { Start: } 2 \text { November } 2012\end{array}$} & \multicolumn{2}{|c|}{$\begin{array}{l}\text { Comparison } 2 \\
\text { Start: } 21 \text { August } 2014\end{array}$} \\
\hline & $\begin{array}{l}\text { Warfarin } \\
(n=35,704)\end{array}$ & $\begin{array}{l}\text { Rivaroxaban } \\
(n=21,064)\end{array}$ & $\begin{array}{l}\text { Warfarin } \\
(n=16,269)\end{array}$ & $\begin{array}{l}\text { Apixaban } \\
(n=5,663)\end{array}$ \\
\hline Age, $y^{b}$ & $64 \pm 16$ & $59 \pm 16$ & $65 \pm 16$ & $64 \pm 16$ \\
\hline Female sex, \% & 51.7 & 49.3 & 52.4 & 52.3 \\
\hline Outpatient treated, \% & 27.9 & 28.5 & 26.7 & 23.9 \\
\hline Pulmonary embolism, \% & 42.5 & 41.3 & 43.6 & 47.7 \\
\hline \multicolumn{5}{|l|}{ Select co-morbidities, \% } \\
\hline Myocardial infarction & 8.5 & 5.3 & 9.5 & 8.9 \\
\hline Heart failure & 18.7 & 11.5 & 20.6 & 20.0 \\
\hline Peripheral artery disease & 16.7 & 10.8 & 18.7 & 16.7 \\
\hline Cerebrovascular disease & 13.5 & 7.9 & 14.3 & 11.8 \\
\hline Dementia & 5.2 & 3.0 & 6.4 & 6.0 \\
\hline Liver disease & 8.8 & 7.5 & 9.3 & 8.6 \\
\hline Diabetes & 27.3 & 20.5 & 28.6 & 28.1 \\
\hline Renal disease & 17.7 & 8.6 & 20.3 & 18.4 \\
\hline Malignancy & 18.5 & 16.4 & 17.8 & 17.3 \\
\hline Cardiac arrhythmia & 32.0 & 24.6 & 34.3 & 33.9 \\
\hline Valvular disease & 17.8 & 13.1 & 19.3 & 19.2 \\
\hline Hypertension & 65.9 & 54.5 & 68.1 & 66.7 \\
\hline Coagulopathy & 10.7 & 8.0 & 12.2 & 10.3 \\
\hline Overweight and obesity & 19.7 & 19.7 & 21.4 & 22.2 \\
\hline Depression & 19.2 & 16.5 & 19.9 & 18.3 \\
\hline \multicolumn{5}{|l|}{ Medications, \% } \\
\hline Insulin & 8.0 & 5.0 & 8.4 & 8.2 \\
\hline Anti-diabetics & 14.4 & 12.2 & 15.2 & 15.5 \\
\hline Anti-hypertensives & 51.5 & 42.0 & 53.8 & 52.9 \\
\hline Statins & 34.7 & 27.7 & 36.6 & 36.4 \\
\hline Anti-platelets & 6.7 & 4.2 & 7.3 & 7.2 \\
\hline Celecoxib & 2.2 & 2.5 & 2.1 & 2.6 \\
\hline Narcotic analgesics & 54.4 & 53.9 & 52.4 & 52.0 \\
\hline Anti-neoplastics & 5.3 & 5.1 & 5.1 & 5.6 \\
\hline
\end{tabular}

Abbreviations: DOAC, direct oral anticoagulant; VTE, venous thromboembolism.

Note: Characteristics of enrolees with a venous thromboembolism diagnosis by initial prescribed oral anticoagulant for DOAC comparisons versus warfarin, OptumLabs Data Warehouse, 2012-2017. ${ }^{\text {. }}$

${ }^{\mathrm{a}}$ Enrolees with an index date before 21 August 2014 were not included in comparisons involving apixaban since it was not approved for treating VTE before this date.

${ }^{\mathrm{b}}$ Mean \pm standard deviation.

concomitant medications, the associations of rivaroxaban and apixaban with lower mortality versus warfarin were no longer present (OR, 0.97, 95\% CI, 0.84, 1.11 for rivaroxaban vs. warfarin; OR, $0.91,95 \%$ CI, 0.72, 1.15 for apixaban vs. warfarin). Associations were similar when the outcome was all-cause mortality within 3 months, although CIs were wider due to fewer events.

Compared with rivaroxaban users, the crude risk of 6-month all-cause mortality was higher among apixaban users (OR, 1.45, $95 \% \mathrm{CI}, 1.19,1.77)$, although this association was attenuated in the adjusted IPTW model (OR, 1.07, 95\% CI, 0.86, 1.34; - Table 2). In sensitivity analyses using only all-cause deaths from the Death Master File, associations were largely similar, although with wide CIs due to a decreased number of events (-Supplementary Table S4, available in the online version).

In - Fig. 1, we summarize the results from this study along with those from the phase III clinical trials and previous observational studies for comparisons of rivaroxaban and apixaban versus VKA therapy. Overall, there was no evidence 
Table 2 Associations between oral anticoagulation and mortality

\begin{tabular}{|c|c|c|c|c|c|c|c|}
\hline & \multirow[t]{3}{*}{$N$} & \multicolumn{2}{|c|}{$N$ all-cause deaths } & \multicolumn{4}{|c|}{ OR $(95 \% \mathrm{Cl})$ for all-cause mortality } \\
\hline & & \multirow[t]{2}{*}{3 months } & \multirow[t]{2}{*}{6 months } & \multicolumn{2}{|l|}{3 months } & \multicolumn{2}{|l|}{6 months } \\
\hline & & & & Crude & IPTW MSM & Crude & IPTW MSM \\
\hline \multicolumn{8}{|c|}{ DOAC vs. warfarin comparisons } \\
\hline Warfarin & 35,704 & 802 & 1,208 & Ref. & Ref. & Ref. & Ref. \\
\hline Rivaroxaban & 21,064 & 299 & 428 & $0.63(0.55,0.72)$ & $0.98(0.83,1.16)$ & $0.59(0.53,0.66)$ & $0.97(0.84,1.11)$ \\
\hline Warfarin & 16,269 & 364 & 545 & Ref. & Ref. & Ref. & Ref. \\
\hline Apixaban & 5,663 & 112 & 155 & $0.88(0.71,1.09)$ & $0.96(0.74,1.26)$ & $0.81(0.68,0.97)$ & $0.91(0.72,1.15)$ \\
\hline \multicolumn{8}{|c|}{ DOAC vs. DOAC comparisons } \\
\hline Rivaroxaban & 14,630 & 202 & 278 & Ref. & Ref. & Ref. & Ref. \\
\hline Apixaban & 5,663 & 112 & 155 & $1.44(1.14,1.82)$ & $1.05(0.81,1.37)$ & $1.45(1.19,1.77)$ & $1.07(0.86,1.34)$ \\
\hline
\end{tabular}

Abbreviations: $\mathrm{Cl}$, confidence interval; DOAC, direct oral anticoagulant; IPTW MSM, inverse probability of treatment weighted marginal structural model; OR, odds ratio.

Note: IPTW weights were estimated using a logistic regression model for treatment, including the covariates age, age, ${ }^{2}$ sex, census region, index date, index date, ${ }^{2}$ diagnosis setting, the constituent Charlson and Elixhauser co-morbidity and frailty variables and concomitant medications. Odds ratios (ORs) and $95 \%$ confidence intervals (Cls) for 3- and 6-month all-cause mortality comparing the use of oral anticoagulants for the treatment of venous thromboembolism, OptumLabs Data Warehouse, 2012-2017.

of a difference in the risk of mortality for most comparisons. Among DVT patients, there was a little evidence suggesting possibly a lower RR of mortality with use of rivaroxaban versus VKA therapy (RR, 0.84, 95\% CI, 0.62, 1.14), although with some heterogeneity $\left(I^{2}=55 \%\right)$ between studies.

Oral anticoagulant use generally remained un-associated with 6-month all-cause mortality in models stratified by VTE type, sex, renal disease, liver disease, overweight and obesity or use of anti-platelet medications (-Fig. 2). For the comparison of apixaban versus warfarin, there was possible evidence of multiplicative interaction with age ( $p$-value for interaction: 0.04 ); the risk of 6-month all-cause mortality was lower among apixaban users compared with warfarin users in those aged less than 65 years (OR, 0.58, 95\% CI, 0.37, 0.90), whereas there was no association in those who were aged 65 years or older

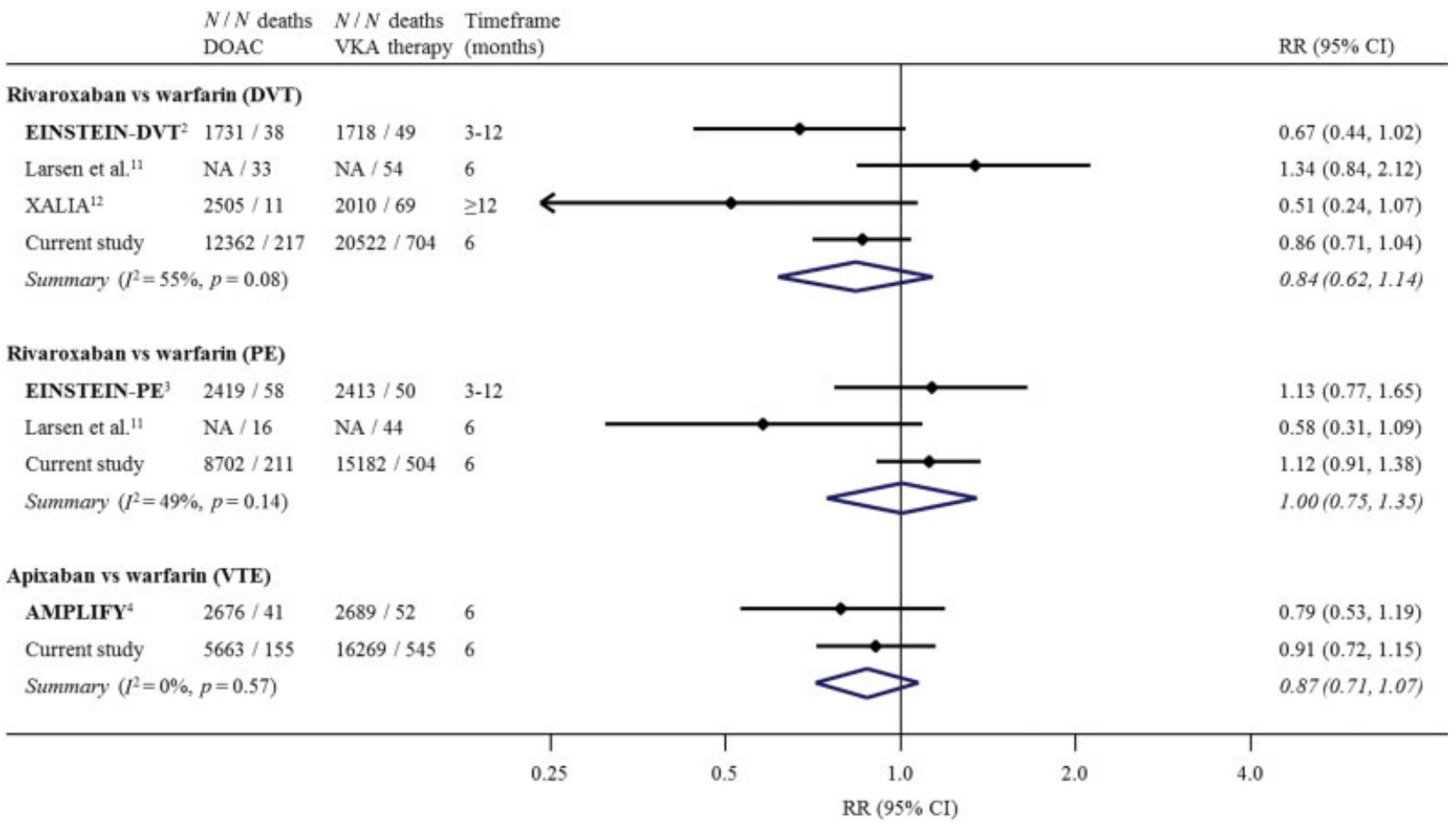

Fig. 1 Meta-analysis of relative risks (RRs) and 95\% confidence intervals ( $\mathrm{Cls}$ ) of all-cause mortality comparing rivaroxaban and apixaban (separately) versus vitamin K antagonist (VKA) therapy for the primary treatment of venous thromboembolism (VTE). Estimates are from phase III clinical trials, ${ }^{2-4}$ previous observational studies ${ }^{11,12}$ and this study. Clinical trials are shown using bold text. Results for rivaroxaban versus VKA therapy are shown stratified by deep vein thrombosis/pulmonary embolism (DVT/PE), in line with the design of the EINSTEIN trials. 
(OR, 1.00, 95\% CI, 0.77, 1.30). However, there was no evidence supporting this interaction on the additive scale (RERI, 0.42, 95\% CI, $-0.44,1.28$ ). Conversely, there was evidence supporting a super-additive interaction with age $\geq 65$ years for the comparison of apixaban versus rivaroxaban (RERI, 0.93), albeit with very wide CIs (95\%
$\mathrm{CI}, 0.01,1.86)$. The risk of 6-month all-cause mortality was higher among apixaban users compared with rivaroxaban users in those aged 65 years or older (OR, 1.24, 95\% CI, 0.95, 1.62 ), whereas the direction of association was the opposite in those aged less than 65 years (OR, 0.75, 95\% CI, 0.48, 1.17 ; $p$-value for multiplicative interaction: 0.06 ).

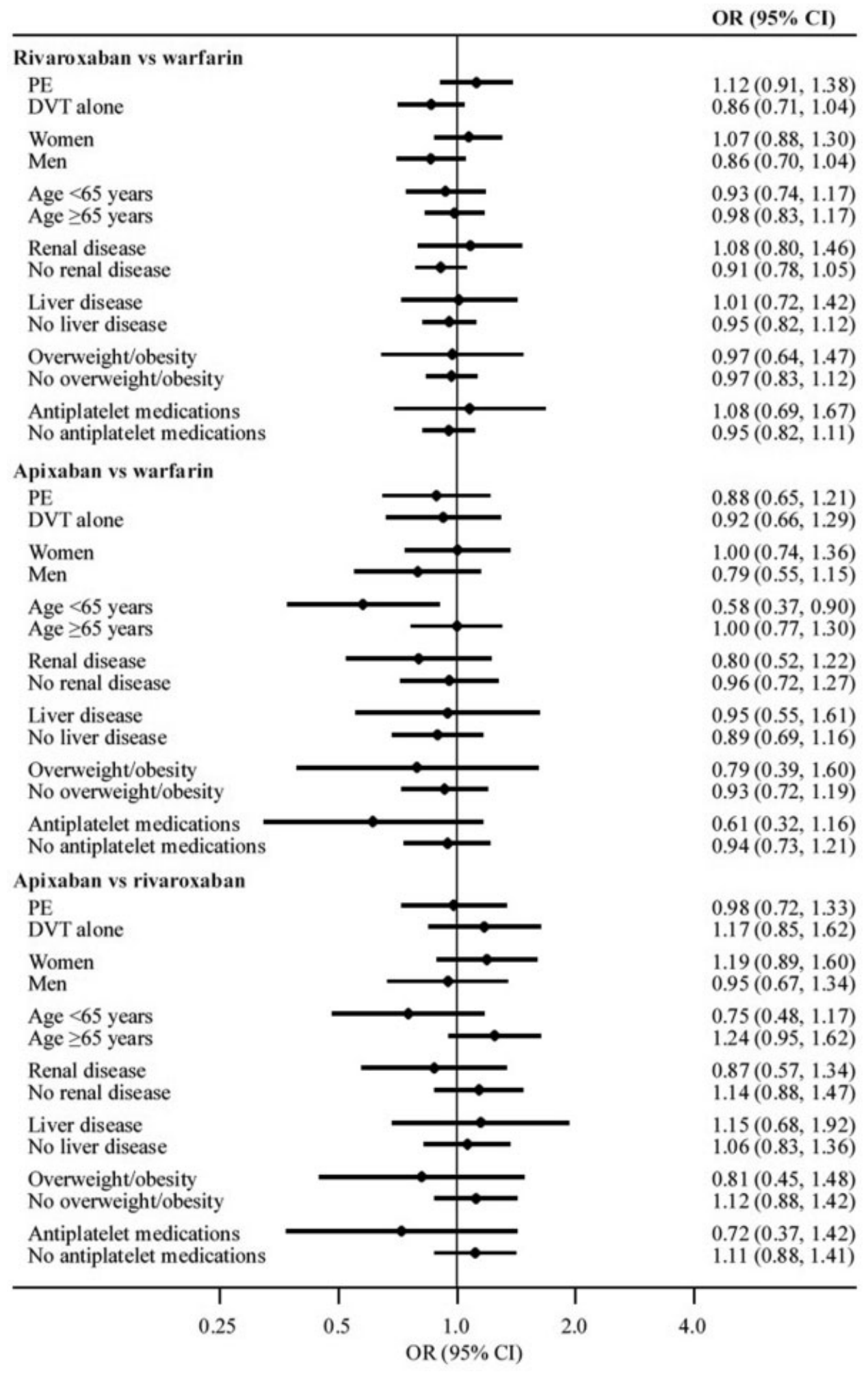

Fig. 2 Stratified odds ratios (ORs) and 95\% confidence intervals ( $\mathrm{Cls}$ ) for 6-month all-cause mortality comparing the use of oral anticoagulants for the treatment of venous thromboembolism. OptumLabs Data Warehouse, 2012-2017. 


\section{Discussion}

In a large administrative dataset, there was no association between the anticoagulant prescribed (warfarin vs. any DOAC) and risk of all-cause mortality during the VTE primary treatment phase (i.e. over the first 3 or 6 months) after adjusting for demographics, co-morbidities and medication use. Similarly, in head-to-head DOAC comparisons, risk of all-cause mortality was similar regardless of the DOAC prescribed. Further, associations generally did not vary when stratified by VTE type, sex, age, co-morbidities or anti-platelet medication use, except for potentially a lower risk of mortality in younger individuals on apixaban versus warfarin or rivaroxaban. These data suggest that other criteria should be used when determining the choice of anticoagulant for the primary treatment of VTE, such as patient preference, cost, recurrent VTE risk or bleeding risk.

This study provides valuable information for assessing the safety of oral anticoagulants with respect to how they have been prescribed in a U.S. routine clinical practice setting over the entire time period through which DOACs have been approved for treating VTE (2012-2017). To our knowledge, this study represents the largest comparison of rivaroxaban versus VKA therapy and apixaban versus VKA therapy with respect to risk of all-cause mortality in terms of total sample size and number of deaths. Our findings, that there were generally no differences in the risk of 3- or 6-month all-cause mortality for VTE patients with prescriptions for rivaroxaban and apixaban in comparison to VKA therapy, are in agreement with the results from the phase III clinical trials and previous observational studies, and our findings have not raised any additional safety risks with DOACs or warfarin in terms of all-cause mortality. ${ }^{1-4,6,11-13}$

Furthermore, this is the first study to compare DOACs in a head-to-head fashion (apixaban vs. rivaroxaban) with respect to risk of all-cause mortality. Our sample size was large for this comparison, allowing us to estimate effect measures with reasonable precision. We did not find any differences in mortality risk for apixaban versus rivaroxaban. While dabigatran and edoxaban are approved for the treatment of VTE, the need for an initial parental treatment phase puts these medications at a great disadvantage to apixaban and rivaroxaban which offer all-oral treatments for VTE. It is unlikely that any advantages of dabigatran and edoxaban (such as lack of metabolism through cytochrome P450 3A4) will outweigh this disadvantage in clinical practice.

Caution should be exercised when interpreting the interaction data with apixaban and age. There is no a priori hypothesis as to why apixaban would have a lower mortality in younger individuals compared with either rivaroxaban or warfarin. While the findings could be true, they may also be due to chance or impacted by unaccounted confounding. After 3 weeks at a higher dose, rivaroxaban is administered once daily to treat VTE, despite having a shorter half-life than apixaban (administered twice daily). More efficient renal metabolism in younger individuals could lead to increased VTE-related mortality. This effect was seen with edoxaban where the efficacy was reduced in individuals with normal renal function in the trial which led to its approval for stroke and systemic embolization prevention in atrial fibrillation. ${ }^{32}$ Compared with warfarin, apixaban is known to have a lower risk of major bleeding. ${ }^{4,33}$ In younger, healthier individuals with fewer risks for death, increased mortality from major bleeding may explain the findings. Further evidence from other study populations is needed to verify this interaction.

In this analysis, there were 583 deaths in total among rivaroxaban and apixaban users, which far exceeds prior observational analyses, each of which had fewer than 183 deaths among DOAC users. $^{11-13}$ However, our analysis, which included mortality information from both the SSA Death Master File and medical claim records, likely misclassified some individuals as living who actually died. The SSA Death Master File is missing up to $40 \%$ of deaths over our study period. ${ }^{22}$ Under the assumption that missed deaths are non-differential with respect to treatment, this type of misclassification should lead to bias towards the null. Further, given the low mortality seen in our analysis, misclassification of some individuals as living who have died would be extremely unlikely to meaningfully impact our results. Captured in-hospital deaths based on medical claim records reduced the severity of this problem. Furthermore, the crude 6-month all-cause death rates in this study were $3.4,2.0$ and $2.7 \%$ among those with prescriptions for warfarin, rivaroxaban and apixaban, respectively. These rates are comparable with the crude all-cause death rates in the EINSTEIN-DVT and EINSTEIN-PE trials (2.2-2.4\% among rivaroxaban and $2.1-2.9 \%$ among VKA users over the treatment period of 3-12 months), ${ }^{2,3}$ the AMPLIFY trial (1.5\% among apixaban and $1.9 \%$ among warfarin users over the 6-month treatment period), ${ }^{4}$ an observational study of unprovoked VTE in Denmark (2.8\% among rivaroxaban and 3.0\% among warfarin users over 6 months $)^{11}$ and a multi-centre observational study of symptomatic DVT $(0.3 \%$ among rivaroxaban and $2.9 \%$ among VKA users over 180 days). ${ }^{12}$

A major limitation of observational research specifically when using claims data is the possibility of uncontrolled confounding (e.g. we cannot adjust for important lifestyle factors such as smoking and physical activity or over-thecounter medications such as aspirin), which can lead to a lack of exchangeability between treatment groups. While the assumption of no uncontrolled confounding is unlikely to be satisfied, we attempted to minimize bias due to confounding by including in our exposure models key demographic, co-morbidity, frailty and medication variables likely to be related to both treatment and mortality. We are reassured that uncontrolled confounding likely has not played a major role in our findings as our results are consistent with and extend results of randomized controlled trials (which randomize to control for confounding), as well as those of smaller observational studies. Further, individuals whose primary treatment for VTE were DOACs tended to be younger and have fewer co-morbid conditions than warfarin users and as such we would expect confounding to favour a lower mortality with DOACs, which we did not see. Additionally, there is no rationale why co-morbid conditions would less likely be documented for DOAC users than warfarin users. While we cannot exclude small differences in mortality by 
primary anticoagulant choice to treat VTE, these are not likely clinically significant based on our results as well as those of other studies.

Another limitation of this study was the limited power for detecting treatment-covariate interactions with respect to the risk of all-cause mortality. In particular, there was a low prevalence $(\leq 20 \%)$ of some co-morbidities of interest, such as renal disease and liver disease, and so the precision of effect estimates was poor in these sub-groups. Future studies should continue to compare the safety of different oral anticoagulants in these specific sub-groups.

Strengths of this study include that it assesses the safety of oral anticoagulants for treatment of VTE in a nationwide sample of private health plan enrolees participating in routine clinical practice, includes a large number of rivaroxaban and apixaban users and is the first study to conduct head-to-head comparisons of DOACs on the risk of all-cause mortality among patients with VTE. Despite the limitations of administrative data, when used with rigorous analytic methods, they represent an efficient way to examine the comparative safety and effectiveness of different treatment strategies. ${ }^{34}$ Randomized trials, in comparison, would need to be very large, and thus prohibitively expensive and time consuming, to capture a similar number of deaths among VTE patients. Furthermore, there is little incentive for drug manufacturers to fund new trials for head-to-head DOAC comparisons. ${ }^{34}$ Non-administrative observational data sources also are typically too small to answer the questions posed here.

In conclusion, in this large sample of private insurance enrolees with a diagnosis claim for VTE and a pharmacy claim for an oral anticoagulant, risk of all-cause mortality was similar for the comparisons of warfarin and the DOACs rivaroxaban, and apixaban, and for head-to-head comparisons of the DOACs. The associations for comparisons of DOACs versus warfarin are in agreement with previous findings from clinical trials and small observational studies, while the findings for head-to-head DOAC comparisons provide new information on the safety of oral anticoagulants for the treatment of VTE.

\section{What is known about this topic?}

- Direct oral anticoagulants are non-inferior to warfarin for preventing recurrent venous thromboembolism.

- Few studies have assessed the comparative effectiveness of oral anticoagulants with respect to risk of allcause mortality.

\section{What does this paper add?}

- Risk of all-cause mortality is similar for warfarin, rivaroxaban and apixaban prescribed for the primary treatment of venous thromboembolism.

- Other criteria such as patient preference, cost, recurrent VTE risk or bleeding risk should be used to determine choice of anticoagulant.

\section{Funding}

This work was supported by National Institutes of Health, National Heart, Lung, and Blood Institute grants R01-HL 122200 (A.A.) and R01-HL131579 (P.L.L.). The funders had no role in the design and conduct of the study; collection, management, analysis and interpretation of the data; preparation, review or approval of the manuscript; and decision to submit the manuscript for publication.

Conflict of Interest

None.

\section{References}

1 Schulman S, Kearon C, Kakkar AK, et al; RE-COVER Study Group. Dabigatran versus warfarin in the treatment of acute venous thromboembolism. N Engl J Med 2009;361(24):2342-2352

2 Bauersachs R, Berkowitz SD, Brenner B, et al; EINSTEIN Investigators. Oral rivaroxaban for symptomatic venous thromboembolism. N Engl J Med 2010;363(26):2499-2510

3 Büller HR, Prins MH, Lensin AW, et al; EINSTEIN-PE Investigators. Oral rivaroxaban for the treatment of symptomatic pulmonary embolism. N Engl J Med 2012;366(14):1287-1297

4 Agnelli G, Buller HR, Cohen A, et al; AMPLIFY Investigators. Oral apixaban for the treatment of acute venous thromboembolism. $\mathrm{N}$ Engl J Med 2013;369(09):799-808

5 Büller HR, Décousus H, Grosso MA, et al; Hokusai-VTE Investigators. Edoxaban versus warfarin for the treatment of symptomatic venous thromboembolism. N Engl J Med 2013;369(15):1406-1415

6 Schulman S, Kakkar AK, Goldhaber SZ, et al; RE-COVER II Trial Investigators. Treatment of acute venous thromboembolism with dabigatran or warfarin and pooled analysis. Circulation 2014;129 (07):764-772

7 Kunz LM, Yeh RW, Normand S-LT. Comparative effectiveness research: does one size fit all? Stat Med 2012;31(25):3062-3065

8 Kearon C, Akl EA, Ornelas J, et al. Antithrombotic therapy for VTE disease: CHEST guideline and expert panel report. Chest 2016; 149(02):315-352

9 Sindet-Pedersen C, Langtved Pallisgaard J, Staerk L, et al. Comparative safety and effectiveness of rivaroxaban versus VKAs in patients with venous thromboembolism. A Danish nationwide registrybased study. Thromb Haemost 2017;117(06):1182-1191

10 Kucher N, Aujesky D, Beer JH, et al. Rivaroxaban for the treatment of venous thromboembolism. The SWIss Venous ThromboEmbolism Registry (SWIVTER). Thromb Haemost 2016;116(03):472-479

11 Larsen TB, Skjøth F, Kjældgaard JN, Lip GYH, Nielsen PB, Søgaard M. Effectiveness and safety of rivaroxaban and warfarin in patients with unprovoked venous thromboembolism: a propensity-matched nationwide cohort study. Lancet Haematol 2017;4 (05):e237-e244

12 Ageno W, Mantovani LG, Haas S, et al. Safety and effectiveness of oral rivaroxaban versus standard anticoagulation for the treatment of symptomatic deep-vein thrombosis (XALIA): an international, prospective, non-interventional study. Lancet Haematol 2016;3(01):e12-e21

13 Jun M, Lix LM, Durand M, et al; Canadian Network for Observational Drug Effect Studies (CNODES) Investigators. Comparative safety of direct oral anticoagulants and warfarin in venous thromboembolism: multicentre, population based, observational study. BMJ 2017;359:j4323

14 OptumLabs. OptumLabs and OptumLabs Data Warehouse (OLDW) Descriptions and Citation. Cambridge, MA: OptumLabs; 2017

15 Kniffin WD Jr, Baron JA, Barrett J, Birkmeyer JD, Anderson FA Jr. The epidemiology of diagnosed pulmonary embolism and deep venous thrombosis in the elderly. Arch Intern Med 1994;154(08):861-866 
16 Cushman M, Tsai AW, White RH, et al. Deep vein thrombosis and pulmonary embolism in two cohorts: the longitudinal investigation of thromboembolism etiology. Am J Med 2004;117(01):19-25

17 Heckbert SR, Kooperberg C, Safford MM, et al. Comparison of selfreport, hospital discharge codes, and adjudication of cardiovascular events in the Women's Health Initiative. Am J Epidemiol 2004;160(12):1152-1158

18 Birman-Deych E, Waterman AD, Yan Y, Nilasena DS, Radford MJ, Gage BF. Accuracy of ICD-9-CM codes for identifying cardiovascular and stroke risk factors. Med Care 2005;43(05):480-485

19 Tamariz L, Harkins T, Nair V. A systematic review of validated methods for identifying venous thromboembolism using administrative and claims data. Pharmacoepidemiol Drug Saf 2012;21 (Suppl 1):154-162

20 Fang MC, Fan D, Sung SH, et al. Validity of using inpatient and outpatient administrative codes to identify acute venous thromboembolism: the CVRN VTE Study. Med Care 2017;55(12): e137-e143

21 Garg RK, Glazer NL, Wiggins KL, et al. Ascertainment of warfarin and aspirin use by medical record review compared with automated pharmacy data. Pharmacoepidemiol Drug Saf 2011;20 (03):313-316

22 da Graca B, Filardo G, Nicewander D. Consequences for healthcare quality and research of the exclusion of records from the Death Master File. Circ Cardiovasc Qual Outcomes 2013;6(01):124-128

23 Quan H, Sundararajan V, Halfon P, et al. Coding algorithms for defining comorbidities in ICD-9-CM and ICD-10 administrative data. Med Care 2005;43(11):1130-1139

$24 \mathrm{Kim} \mathrm{DH}$, Schneeweiss S. Measuring frailty using claims data for pharmacoepidemiologic studies of mortality in older adults: evidence and recommendations. Pharmacoepidemiol Drug Saf 2014;23(09):891-901

25 Ray WA. Evaluating medication effects outside of clinical trials: new-user designs. Am J Epidemiol 2003;158(09):915-920

26 Hernán MA, Alonso A, Logan R, et al. Observational studies analyzed like randomized experiments: an application to postmenopausal hormone therapy and coronary heart disease. Epidemiology 2008;19(06):766-779

27 Robins JM, Hernán MA, Brumback B. Marginal structural models and causal inference in epidemiology. Epidemiology 2000;11 (05):550-560

28 Alonso A, Bengtson LGS, MacLehose RF, Lutsey PL, Chen LY, Lakshminarayan K. Intracranial hemorrhage mortality in atrial fibrillation patients treated with dabigatran or warfarin. Stroke 2014;45(08):2286-2291

29 Funk MJ, Landi SN. Misclassification in administrative claims data: quantifying the impact on treatment effect estimates. Curr Epidemiol Rep 2014;1(04):175-185

30 Hosmer DW, Lemeshow S. Confidence interval estimation of interaction. Epidemiology 1992;3(05):452-456

31 VanderWeele TJ, Knol MJ. A tutorial on interaction. Epidemiol Methods 2014;3:33-72

32 Bohula EA, Giugliano RP, Ruff CT, et al. Impact of renal function on outcomes with edoxaban in the ENGAGE AF-TIMI 48 Trial. Circulation 2016;134(01):24-36

33 Granger CB, Alexander JH, McMurray JJV, et al; ARISTOTLE Committees and Investigators. Apixaban versus warfarin in patients with atrial fibrillation. N Engl J Med 2011;365(11):981-992

34 Parks AL, Redberg RF. Dabigatran compared with rivaroxaban vs warfarin-reply. JAMA Intern Med 2017;177(05):744 